\title{
Second-Order Nonlinearity Assisted by Dual Surface Plasmon Resonance Modes in Perforated Gold Film
}

\author{
Renlong Zhou, Guozheng Nie, Lingxi Wu, Qiong Liu, \\ Suxia Xie, Mengxiong Wu, Lisan Zeng, and Yingyi Xiao \\ School of Physics and Electronic Science, Hunan University of Science and Technology, Xiangtan 411201, China \\ Correspondence should be addressed to Renlong Zhou; rlzhou@mail.sitp.ac.cn
}

Received 29 November 2013; Accepted 12 January 2014; Published 20 February 2014

Academic Editor: Wen Lei

Copyright (c) 2014 Renlong Zhou et al. This is an open access article distributed under the Creative Commons Attribution License, which permits unrestricted use, distribution, and reproduction in any medium, provided the original work is properly cited.

\begin{abstract}
We have studied analytically the reflection assisted with surface plasmon through the square lattice perforated gold film. Under the excitation of the external electromagnetic field with one or two different frequencies, the second-order nonlinearity exists in this noncentrosymmetric metal-based metamaterial. We employed the two surface plasmon resonances modes with different lattice periods. With the excitation of two different plasmon resonances modes, the strong local field induces an expected increase of the second-order nonlinearity including second harmonic generation as well as the sum (difference) frequency generation. The field distributions results also indicate that the enhancement of sum frequency signals and difference frequency signals strongly depends on surface plasmon resonance effect.
\end{abstract}

\section{Introduction}

The optical second harmonic generation (SHG) was first observed for the one-dimensional metal surfaces in 1965 [1]. The optical transmission of light through metal film has been shown to be orders of magnitude higher than that expected by the classical aperture theory due to the surface plasmon (SP) resonances [2-5]. The SHG in such a perforated metal film with different topographical nanoscale apertures has also been the focus of many researchers $[6,7]$. Zeng et al. has found that the SHG effect mainly results from the convective derivative of the continuous electron current [7]. The enhanced linear fundamental field gives rise to the local SHG at the tip surface, thereby creating a highly confined photon source of SHG. The SHG efficiency has been enhanced in a circularly symmetric structure. It has been shown that the SHG is a symmetry-sensitive process. The arrangement of the nanoholes in a random way can break out the symmetry and has been found to favor SHG [8]. In addition, the less-symmetric double-hole array has been studied experimentally and found to cause the enhancement of SHG when the sharp tips are formed by folding the double holes [6]. The SHG has been also investigated in geometric configurations such as split-ring resonators, T-shaped, and Lshaped nanoparticles [9-13].

For the ideally infinite metal surfaces, it is well known that the dominant second harmonic electric dipole source occurs only at the interface between centrosymmetric media, in which the inversion symmetry is broken. The higher order multipole sources merely provide a relatively small bulk SHG polarization density. On the other hand, for low-symmetric or even asymmetric nanoparticles, such as gold split-ring resonators, SHG dipolar polarizability may be presented in the whole volume and not limited at the interface. Consequently, the overall shape of the nanoparticles plays a significant role in determining the second harmonic response $[14,15]$. In this paper, we have investigated the SHG, sum frequency generation (SFG), and difference frequency generation (DFG) from gold film with a periodic subwavelength air nanohole patterns by means of the three-dimensional (3D) finite-difference time-domain (FDTD) algorithm. The enhanced linear fundament field causes the local secondorder nonlinearity. Thereby the local SFG, DFG, and SHG can act as highly confined photon sources. The main results are shown as follows: (1) the reflection of the fundamental light results from the enhancement of the local field due to the SP 
resonance effect and the strong local field induces an expected increase of second-order nonlinearity; (2) the electric field distributions of fundamental light and the nonlinear signals are different for different SP resonance modes.

\section{Structure and Calculation Method}

In the second-order nonlinearity of gold nanostructures, the classical theoretical method of the second-order nonlinearity calculation has been proposed in [7]. The interaction between light and Drude-type metal is generally described by the time-dependent Maxwell equations which are coupled to an equation that describes the light induced oscillation of quasifree electrons in the metal. The linear response of the gold nanostructures is given by [15]:

$$
\begin{gathered}
\frac{\partial B^{(1)}}{\partial t}=-\nabla \times E^{(1)}, \\
\frac{\partial E^{(1)}}{\partial t}=c^{2} \nabla \times B^{(1)}-\frac{1}{\varepsilon_{0}} j^{(1)}, \\
j^{(1)}=-i \omega p^{(1)}=-i \omega \varepsilon_{0}\left(\varepsilon_{r}-1\right) E^{(1)}, \\
\frac{\partial j^{(1)}}{\partial t}=-\gamma j^{(1)}+\frac{e^{2} n_{0}}{m_{e}} E^{(1)} .
\end{gathered}
$$

The second-order nonlinearity of the gold film can be considered as follows:

$$
\begin{gathered}
\frac{\partial B^{(2)}}{\partial t}=-\nabla \times E^{(2)}, \\
\frac{\partial E^{(2)}}{\partial t}=c^{2} \nabla \times B^{(2)}-\frac{1}{\varepsilon_{0}} j^{(2)}, \\
j^{(2)}=-i \omega p^{(2)}=-i \omega \varepsilon_{0}\left(\varepsilon_{r}-1\right) E^{(2)}+S^{(2)}, \\
\frac{\partial j^{(2)}}{\partial t}=-\gamma j^{(2)}+\frac{e^{2} n_{0}}{m_{e}} E^{(2)}+S^{(2)}, \\
S^{(2)}=\sum_{k} \frac{\partial}{\partial r_{k}}\left(\frac{j^{(1)} j^{(1)}}{e n_{0}}\right) \\
-\frac{e}{m_{e}}\left[\varepsilon_{0}\left(\nabla \cdot E^{(1)}\right) E^{(1)}+j^{(1)} \times B^{(1)}\right] .
\end{gathered}
$$

Here, $k$ represents the $x, y$, and $z$ coordinates. $J^{(1)}$ and $J^{(2)}$ represent the current density vectors of fundamental and harmonic waves, respectively. $E^{(1)}$ and $E^{(2)}, B^{(1)}$ and $B^{(2)}$ are the electric field and magnetic flux intensity vectors of fundamental and harmonic waves, respectively. $n_{0}$ is the ion density, $m_{e}$ is the electron mass, $c$ the light speed in vacuum air, $e$ is the elementary charge, and $S^{(2)}$ is the nonlinear source of the plasma for second-order nonlinearity, respectively. It includes three terms of different physical mechanisms. The first term represents a generalized divergence originating from the convective time derivative of the electron velocity field. The second and third terms are the electric and magnetic components of the Lorentz force, respectively [7].

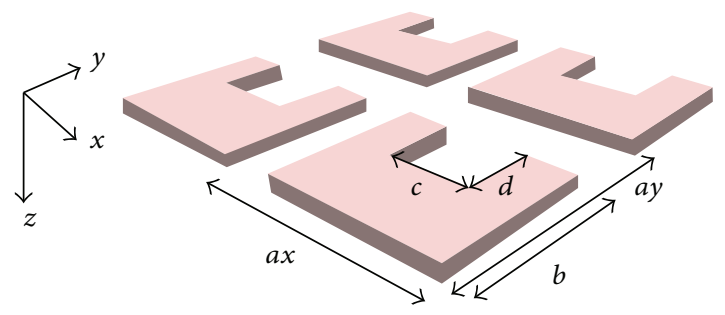

FIGURE 1: The structure of a perforated gold film with the thickness $h=30.5 \mathrm{~nm}$ and the lattice periods $a=a x=a y=305 \mathrm{~nm}$. There has the unit cell shape with $b=219 \mathrm{~nm}, c=131 \mathrm{~nm}$, and $d=97 \mathrm{~nm}$. The input light wave is polarized along the $x$ direction and propagates along the $z$ direction. form

The permittivity $\varepsilon_{r}(\omega)$ of the gold nanostructures has the

$$
\varepsilon_{r}(\omega)=1-\frac{\omega_{p}^{2}}{\omega(\omega+i \gamma)} .
$$

In the expression, the $\gamma$ is the phenomenological collision frequency, and $w_{p}=\sqrt{e^{2} n_{0} / m_{e} \varepsilon_{0}}$ is the bulk plasma frequency according to the well-known Drude model of gold. The bulk plasma frequency and phenomenological collision frequency of gold are taken as $w_{p}=1.367 \times 10^{16} \mathrm{~s}^{-1}$ and $\gamma=6.478 \times 10^{13} \mathrm{~s}^{-1}$, respectively. The permittivity $\varepsilon_{r}(\omega)$ can become negative at frequencies below $\omega_{p}$. The $n_{0}$ is the charge carrier concentration; $m$ is the effective mass of the charge carriers.

The FDTD approach is applied for the numerical calculation of the above first-order and second-order equations. There are two computational loops for the calculations of the fundamental and second harmonic fields in the program. Yee's discretization scheme is utilized so that all electric and magnetic components can be defined in a cubic grid. The fields are temporally separated by a half time step and spatially interlaced by a half grid cell. The perfectly matched absorbing boundary conditions are employed at the below and top of the computational space along the $z$ direction, and the periodic boundary conditions are used on the boundaries of $x$ and $y$ directions. Only one unit cell of the periodic holes array is considered in our computational space.

The structure of a perforated gold film is shown in Figure 1 . The incident wave, polarized along the $x$ direction, propagates along the $z$-axis which is generated by a total field/scattering field technique. Then, we calculate the temporal reflection of the fundamental light and second-order nonlinearity by considering the normal incident light on the perforated semiconductor film.

In our calculation, the size of the spatial cell is set as $3.05 \mathrm{~nm}$. We consider the structure of a perforated gold film with the thickness $h=30.5 \mathrm{~nm}$. The structure of a perforated gold film has a lattice constant $a=305 \mathrm{~nm}$. The lattice constant is $a x=a y=305 \mathrm{~nm}$ along the $x$-axis and $y$-axis directions, respectively. There has the unit cell shape with $b=219 \mathrm{~nm}, c=131 \mathrm{~nm}$, and $d=97 \mathrm{~nm}$. Each unit of the perforated gold film is noncentrosymmetric as shown 


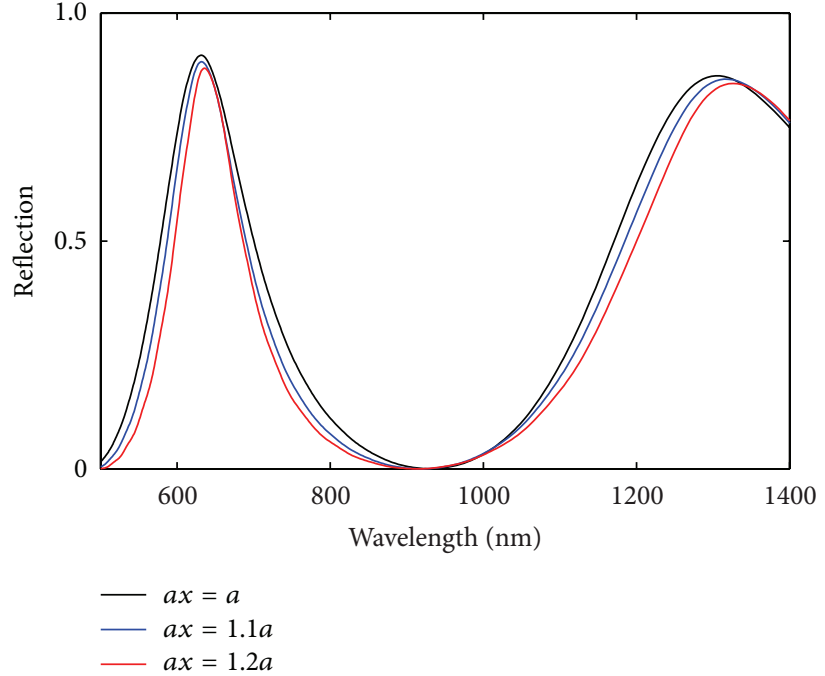

(a)

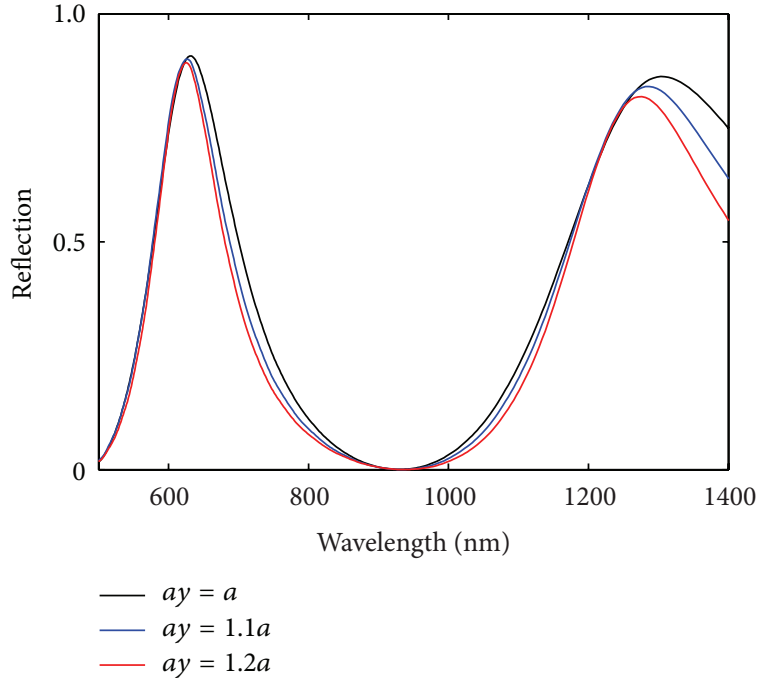

(b)

FIGURE 2: Reflection of FFW through a square lattice perforated gold film for lattice constant (a) along $x$ direction $a x=a$ (black line), $1.1 a$ (blue line), and $1.2 a$ (red line), (b) along y direction $a y=a$ (black line), $1.1 a$ (blue line), and $1.2 a$ (red line), respectively.

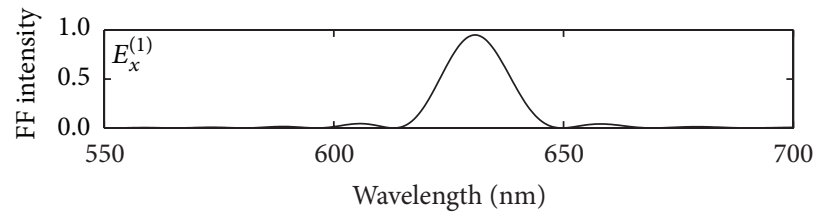

(a)

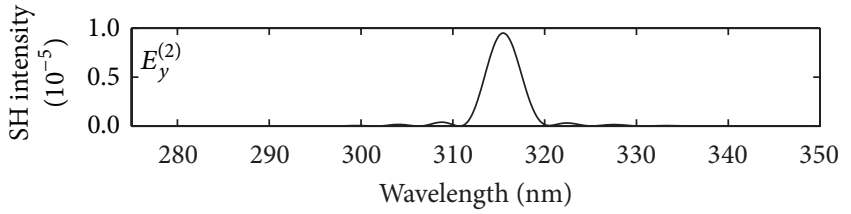

(b)

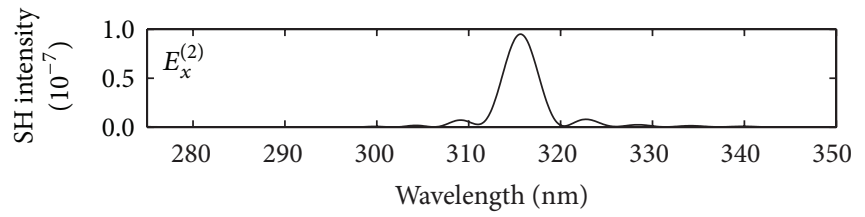

(c)

Figure 3: (a) The spectrum for $E_{x}^{(1)}$ component of FFW with wavelength $630 \mathrm{~nm}$. The spectrum for (b) $E_{y}^{(2)}$ and (c) $E_{x}^{(2)}$ component of SHG with wavelength $315 \mathrm{~nm}$.

in Figure 1. The input plane wave is polarized along the $x$ direction by exciting a plane of identical dipoles in phase and propagates along the $z$ direction.

\section{Results and Discussions}

First, the normalized reflection of fundamental frequency wave (FFW) through the square lattice perforated gold film for different lattice constants along $x$ direction $a x=a$ (black line), $1.1 a$ (blue line), and $1.2 a$ (red line) is investigated here, and the calculation results are shown in Figure 2(a). There are two different SP resonance modes at the wavelengths $630 \mathrm{~nm}$ and $1295 \mathrm{~nm}$ for lattice constants $a x=a$ (black line). When the lattice constant along $x$ direction increases while the holes shape is fixed, the short-wavelength SP resonance mode does not move significantly while the long-wavelength SP resonance mode is blue-shifted dramatically. The normalized fundamental frequency wave reflection for different lattice constants along y direction $a y=1.0 a, 1.1 a$, and $1.2 a$ is also shown in Figure 2(b). When the lattice constant along the $y$ direction increases, the short-wavelength SP resonance mode does not move significantly while the long-wavelength SP resonance mode red-shifts slightly.

To obtain the SHG of the gold film with a periodic subwavelength air nanohole patterns, the incident FFW $E_{x}^{(1)}$ with wavelengths $\lambda$ has the form $E_{x}^{(1)}=E_{0} \sin (2 \pi c t / \lambda)$, where $E_{0}$ is amplitude.

Under the continuous incident FFW $E_{x}^{(1)}$ with wavelengths $\lambda=630 \mathrm{~nm}$, one can see spectrum of FFW with the resonance wavelength $630 \mathrm{~nm}$ in Figure 3(a). It is also shown that the SHG spectrum of the $E_{y}^{(2)}$ and $E_{x}^{(2)}$ with the wavelength $315 \mathrm{~nm}$ is shown in Figures 3(b)-3(c), 


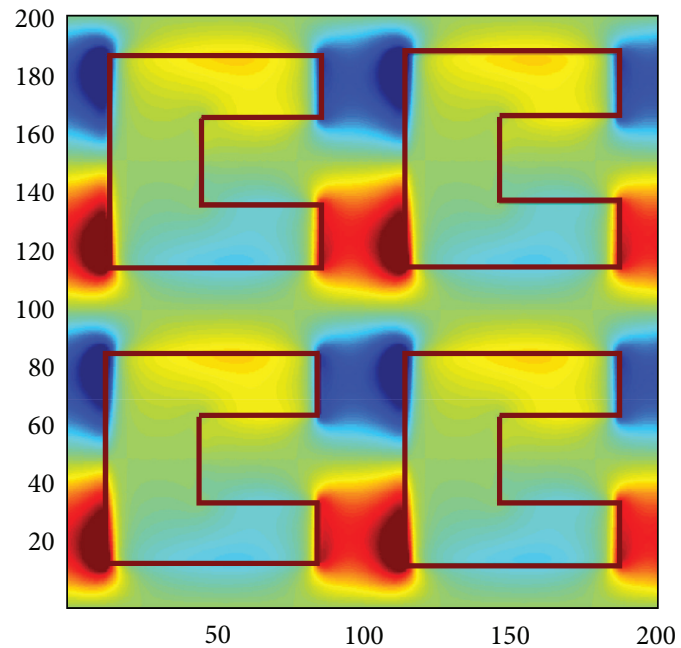

(a) $E_{x}^{(1)}, 630 \mathrm{~nm}$

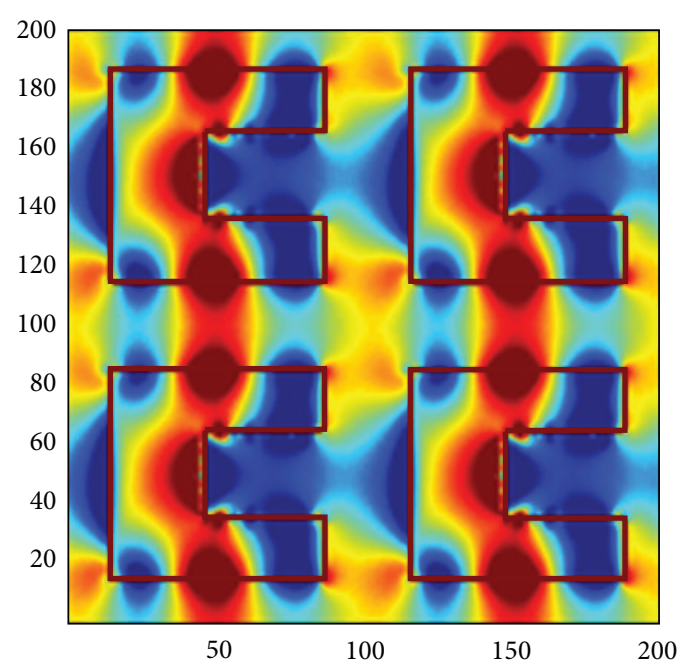

(c) $E_{x}^{(2)}, 315 \mathrm{~nm}$

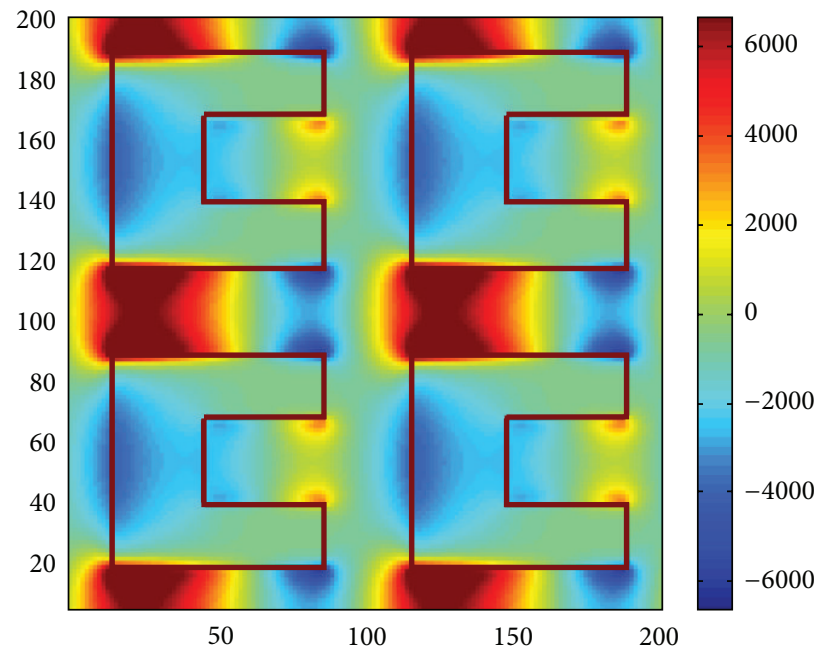

(b) $E_{y}^{(1)}, 630 \mathrm{~nm}$

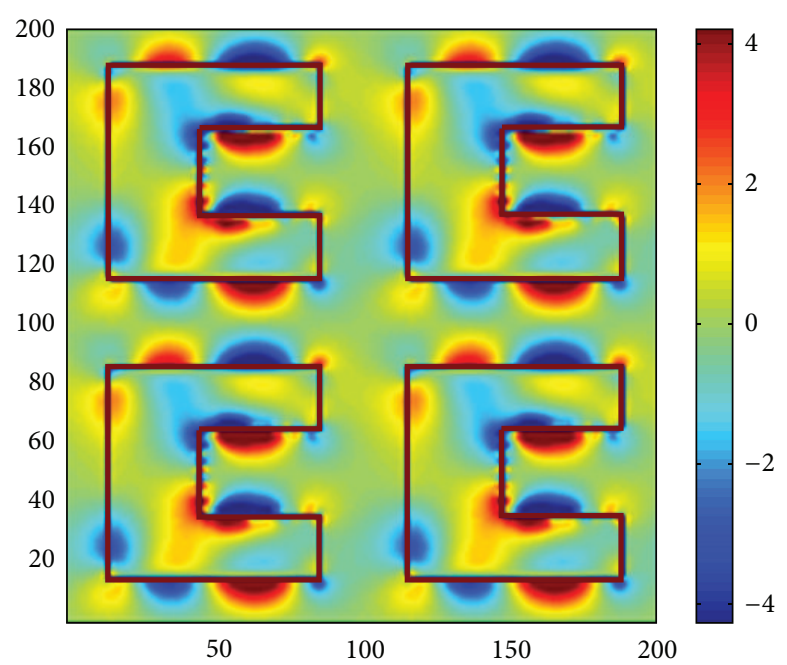

(d) $E_{y}^{(2)}, 315 \mathrm{~nm}$

FIGURE 4: The electric field distribution of FFW (a) $E_{x}^{(1)}$ and (b) $E_{y}^{(1)}$, and electric field distribution of SHG (c) $E_{x}^{(2)}$ and (d) $E_{y}^{(2)}$ at wavelengths $630 \mathrm{~nm}$ and $315 \mathrm{~nm}$, respectively.

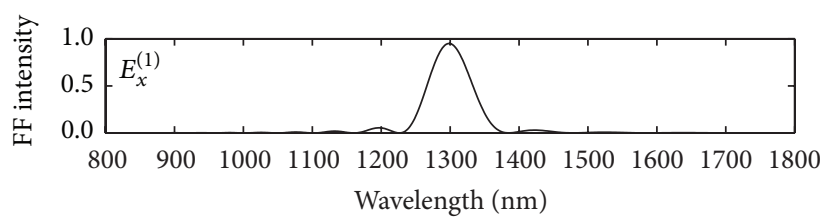

(a)

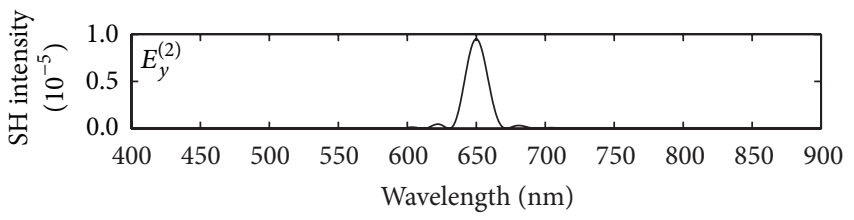

(b)

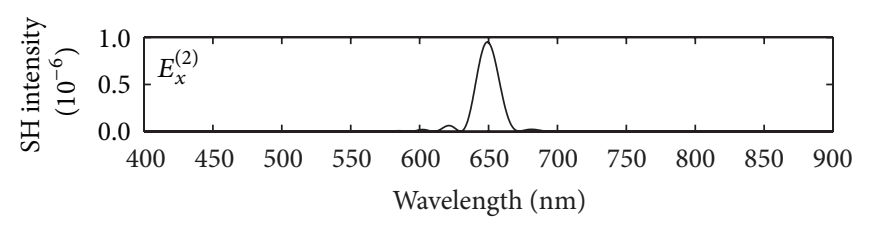

(c)

Figure 5: (a) The spectrum for $E_{x}^{(1)}$ component of fundamental frequency wave at the wavelength $1295 \mathrm{~nm}$. Spectrum for (b) $E_{y}^{(2)}$ and (c) $E_{x}^{(2)}$ component of SHG at the wavelength $648 \mathrm{~nm}$. 


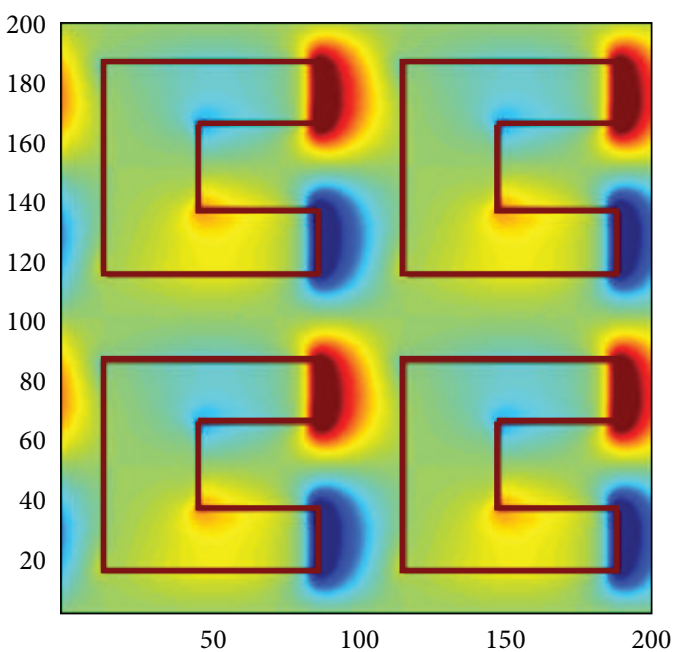

(a) $E_{x}^{(1)}, 1295 \mathrm{~nm}$

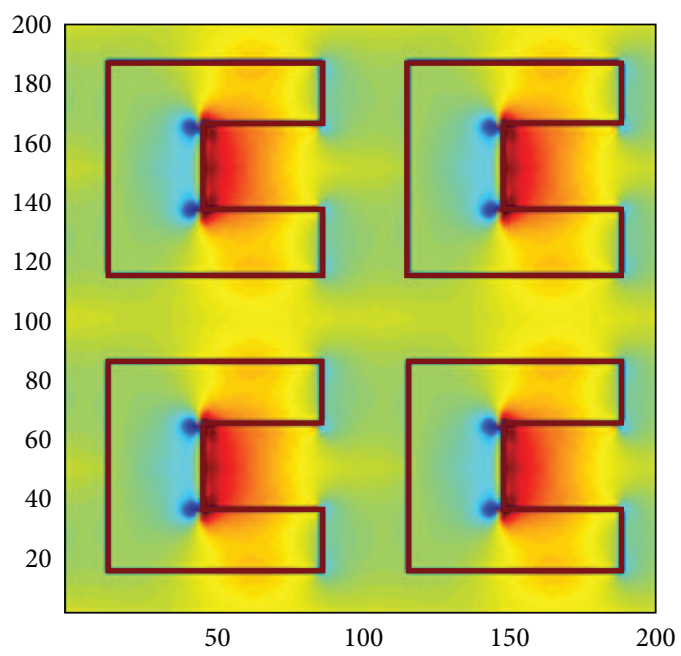

(c) $E_{x}^{(2)}, 648 \mathrm{~nm}$

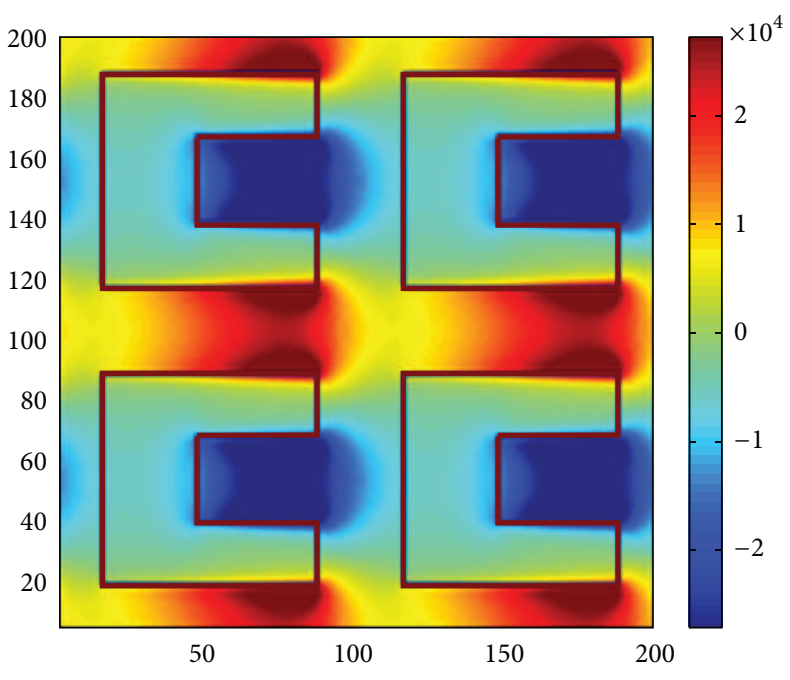

(b) $E_{y}^{(1)}, 1295 \mathrm{~nm}$

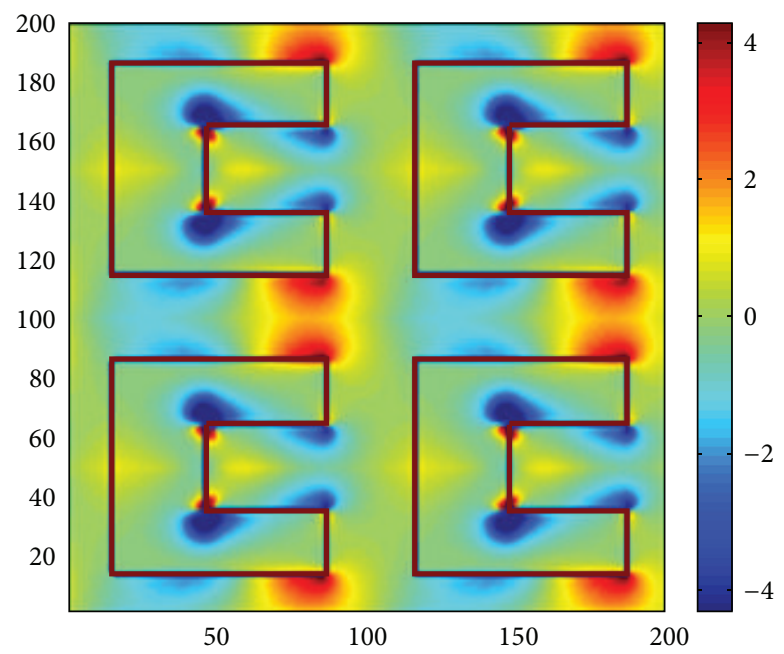

(d) $E_{y}^{(2)}, 648 \mathrm{~nm}$

Figure 6: The electric field distribution of fundamental frequency field and second harmonic field above the gold film region at wavelengths $1295 \mathrm{~nm}$ and $648 \mathrm{~nm}$, respectively.

respectively. The SHG conversion efficiencies $\eta$ are defined in the second-order nonlinear optical process as the expression $\left|E^{(2)}\left(2 \omega_{0}\right) / E^{(1)}\left(\omega_{0}\right)\right|^{2}$. Where $\omega_{0}$ is the frequency of the incident FFW. The $y$-polarized SHG conversion efficiencies are about $10^{-5}$ while the $x$-polarized SHG conversion efficiencies is about $10^{-7}$ for the FFW at the wavelength $630 \mathrm{~nm}$ as shown in Figures 3(b)-3(c).

The transmission of the FFW results from an enhancement of the local field. The strong local field and noncentrosymmetry induces an increase of second harmonic nonlinearity signals. The electric field distribution of $E_{x}^{(1)}$ and $E_{y}^{(1)}$ for FFW above the gold film region at wavelengths $630 \mathrm{~nm}$ is also shown in Figures 4(a)-4(b), respectively. And the electric field distribution of $E_{x}^{(2)}$ and $E_{y}^{(2)}$ for SHG above the gold film region at wavelengths $315 \mathrm{~nm}$ is also shown in Figures $4(\mathrm{c})-4(\mathrm{~d})$. It is noted that the different distribution of fundamental frequency and second harmonic is shown in Figures $4(a)-4(d)$.

Under the continuous incident wave $E_{x}^{(1)}$ with wavelengths $\lambda=1295 \mathrm{~nm}$, one can see spectrum of FFW at wavelength $648 \mathrm{~nm}$ is in Figure 5(a). It is also shown that the spectrum of the $E_{y}^{(2)}$ and the $E_{x}^{(2)}$ component of SHG at wavelength $648 \mathrm{~nm}$ in Figures 5(b)-5(c), respectively. The SHG conversion efficiencies for $y$ polarization are about $10^{-5}$ while they are about $10^{-6}$ for $x$ polarization at wavelength $1295 \mathrm{~nm}$ in Figures 6(b)-6(c). The electric field distribution of $E_{x}^{(1)}$ and $E_{y}^{(1)}$ for FFW above the gold film region at wavelengths $1295 \mathrm{~nm}$ is also shown in Figures 6(a)-6(b), respectively. The electric field distribution of $E_{x}^{(2)}$ and $E_{y}^{(2)}$ for SHG above the gold film region at wavelengths $648 \mathrm{~nm}$ is also shown in Figures 6(c)-6(d). 


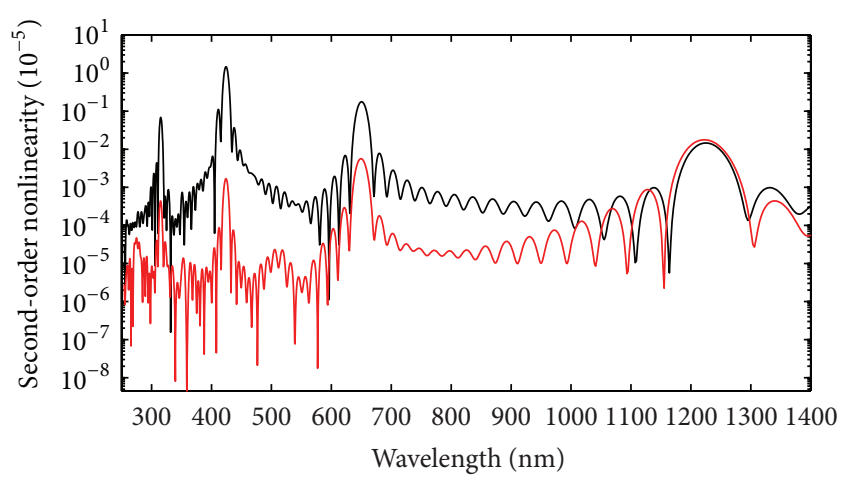

$-E_{y}^{(2)}$
$-E_{x}^{(2)}$

FIgURE 7: The spectrum of second-order nonlinearity signals at the wavelength $315 \mathrm{~nm}, 424 \mathrm{~nm}, 650 \mathrm{~nm}$, and $1226 \mathrm{~nm}$ for the continuous incident wave $E_{x}^{(1)}$ with wavelengths $\lambda_{1}=630 \mathrm{~nm}$ and $\lambda_{2}=1295 \mathrm{~nm}$, respectively.

Under the continuous incident wave $E_{x}^{(1)}$ with two different wavelengths $\lambda_{1}=630 \mathrm{~nm}$ and $\lambda_{2}=1295 \mathrm{~nm}, E_{x}=$ $E_{0} \sin \left(2 \pi c t / \lambda_{1}\right)+E_{0} \sin \left(2 \pi c t / \lambda_{2}\right)$, we can obtain the secondorder nonlinearity including SHG as well as the sum (difference) frequency generation.

When continuous wave $E_{x}^{(1)}$ with two different wavelengths $630 \mathrm{~nm}$ and $1295 \mathrm{~nm}$ is incident, it is noted that there are four peaks for second-order nonlinearity at the wavelengths $315 \mathrm{~nm}, 424 \mathrm{~nm}, 650 \mathrm{~nm}$, and $1226 \mathrm{~nm}$ in Figure 7, respectively. We find that the second-order nonlinearity modes at wavelengths $315 \mathrm{~nm}$ and $650 \mathrm{~nm}$ are obtained from the two incident continuous waves with wavelengths $630 \mathrm{~nm}$ and $1295 \mathrm{~nm}$ due to the SHG effect, respectively. The secondorder nonlinearity mode at wavelength $424 \mathrm{~nm}$ is the sum frequency field signals for two incident continuous waves with wavelengths $630 \mathrm{~nm}$ and $1295 \mathrm{~nm}$. And the SHG conversion efficiencies of sum frequency field signal for two FFW incidences are about $10^{-5}$. The second-order nonlinearity mode at wavelength $1226 \mathrm{~nm}$ is the difference frequency field for two FFW waves with wavelengths $630 \mathrm{~nm}$ and $1295 \mathrm{~nm}$. The transmission of the fundamental light results from an enhancement of the local field. The strong local field induces an increase of the four second-order nonlinearity signals.

According to the fundamental wave field and the secondorder harmonic field analysis, it can be found that a significant interaction of light with the perforated gold film is the strong enhancement of localized field amplitude due to the SP resonance effect. The light propagation through the holes results in the enhancement of the second-order nonlinearity including second harmonic generation as well as the sum (difference) frequency generation.

\section{Conclusions}

We have investigated transmission assisted with surface plasmon through a perforated gold film. The enhanced transmission of the fundamental light results from an enhancement of the local field. The strong local field induces a desired increase of the second-order nonlinearity including second harmonic generation as well as the sum (difference) frequency generation signals. The field distributions results also indicate that the enhancement of difference frequency signals strongly depends on the SP resonance effect. Importantly, we find that the SHG conversion efficiencies of SHG signal for one fundamental frequency wave incidence are about $10^{-5}$. And we also find that the SHG conversion efficiencies of sum frequency field signal for two fundamental frequency wave incidences are about $10^{-5}$.

\section{Conflict of Interests}

The authors declare that there is no conflict of interests regarding the publication of this paper.

\section{Acknowledgments}

This work was supported by the National Natural Science Foundation of China under Grant $(11247003,51175172)$ and Scientific Research Fund of Hunan Provincial Education Department (13C323). The authors would especially like to thank Dr. Yong Zeng of University of Arizona for the discussion of FDTD simulations.

\section{References}

[1] F. Brown, R. E. Parks, and A. M. Sleeper, "Nonlinear optical reflection from a metallic boundary," Physical Review Letters, vol. 14, no. 25, pp. 1029-1031, 1965.

[2] N. Guo, W. D. Hu, X. S. Chen, L. Wang, and W. Lu, "Enhanced plasmonic resonant excitation in a grating gated field-effect transistor with supplemental gates," Optics Express, vol. 21, no. 2, pp. 1606-1614, 2013.

[3] L. Wang, W. Hu, J. Wang et al., "Plasmon resonant excitation in grating-gated AlN barrier transistors at terahertz frequency," Applied Physics Letters, vol. 100, no. 12, Article ID 123501, 2012.

[4] W. D. Hu, L. Wang, X. S. Chen et al., "Room-temperature plasmonic resonant absorption for grating-gate GaN HEMTs in far infrared terahertz domain," Optical and Quantum Electronics, vol. 45, no. 7, pp. 713-720, 2013.

[5] G. Li, X. Chen, B. Ni et al., "Fractal H-shaped plasmonic nanocavity," Nanotechnology, vol. 24, no. 20, Article ID 205702, 2013.

[6] G. Li, X. Chen, O. Li et al., "A novel plasmonic resonance sensor based on an infrared perfect absorber," Journal of Physics D, vol. 45, no. 20, Article ID 205102, 2012.

[7] Y. Zeng, W. Hoyer, J. Liu, S. W. Koch, and J. V. Moloney, "Classical theory for second-harmonic generation from metallic nanoparticles," Physical Review B, vol. 79, no. 23, Article ID 235109, 2009.

[8] A. Nahata, R. A. Linke, T. Ishi, and K. Ohashi, "Enhanced nonlinear optical conversion from a periodically nanostructured metal film," Optics Letters, vol. 28, no. 6, pp. 423-425, 2003.

[9] F. B. P. Niesler, N. Feth, S. Linden et al., "Second-harmonic generation from split-ring resonators on a GaAs substrate," Optics Letters, vol. 34, no. 13, pp. 1997-1999, 2009.

[10] M. W. Klein, M. Wegener, N. Feth, and S. Linden, "Experiments on second- and third-harmonic generation from magnetic 
metamaterials: erratum," Optics Express, vol. 16, no. 11, p. 8055, 2008.

[11] M. W. Klein, C. Enkrich, M. Wegener, and S. Linden, "Secondharmonic generation from magnetic metamaterials," Science, vol. 313, no. 5786, pp. 502-504, 2006.

[12] S. Kujala, B. K. Canfield, M. Kauranen, Y. Svirko, and J. Turunen, "Multipole interference in the second-harmonic optical radiation from gold nanoparticles," Physical Review Letters, vol. 98, no. 16, Article ID 167403, 2007.

[13] B. K. Canfield, H. Husu, J. Laukkanen et al., "Local field asymmetry drives second-harmonic generation in noncentrosymmetric nanodimers," Nano Letters, vol. 7, no. 5, pp. 12511255, 2007.

[14] J. Nappa, G. Revillod, I. Russier-Antoine, E. Benichou, C. Jonin, and P. F. Brevet, "Electric dipole origin of the second harmonic generation of small metallic particles," Physical Review B, vol. 71, no. 16, Article ID 165407, 2005.

[15] Y. Zeng and J. V. Moloney, "Volume electric dipole origin of second-harmonic generation from metallic membrane with none entro symmetric patterns," Optics Letters, vol. 34, no. 18, pp. 2844-2846, 2009. 

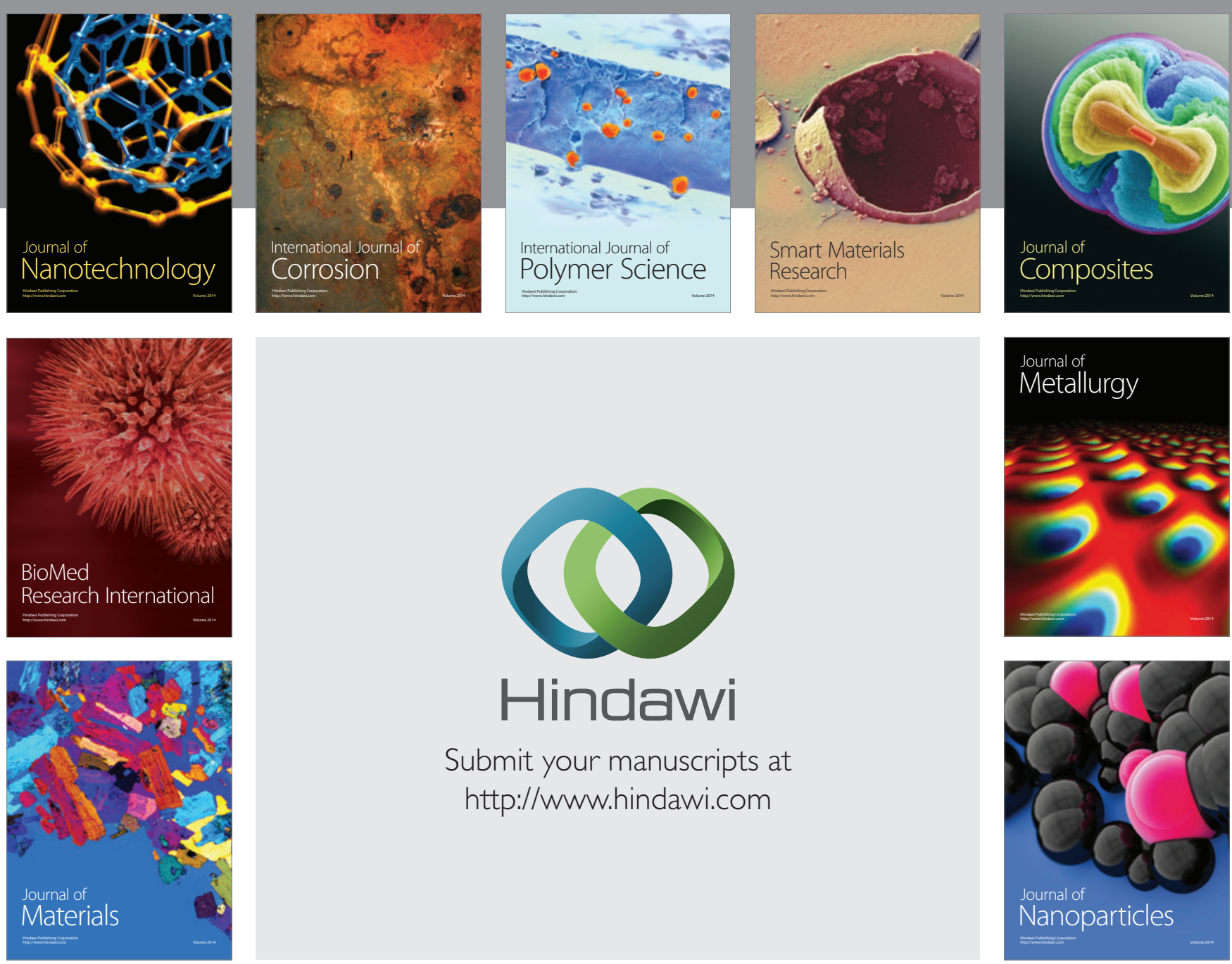

Submit your manuscripts at http://www.hindawi.com
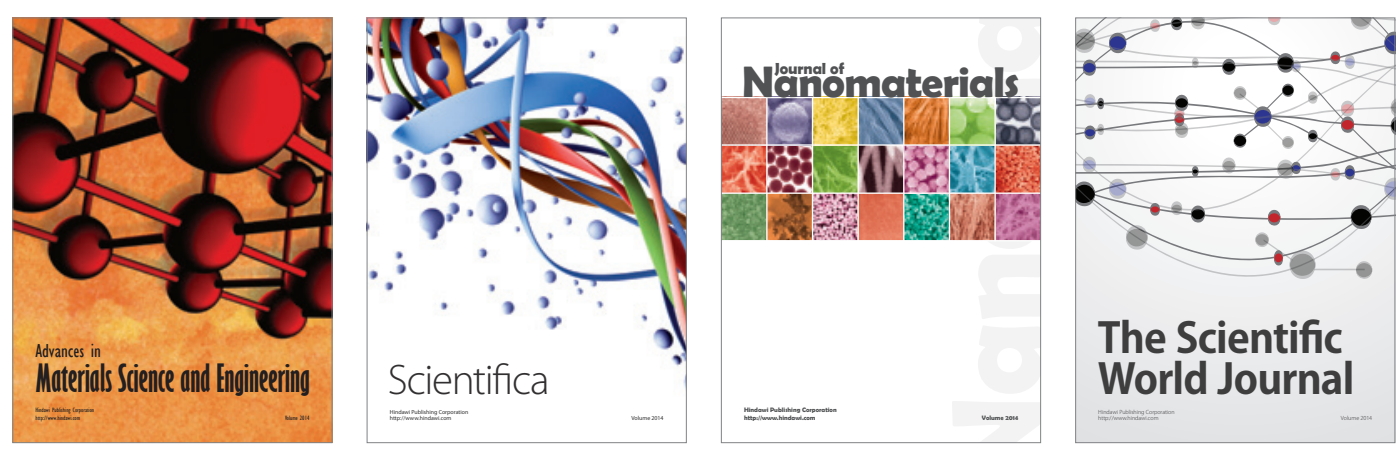

\section{The Scientific World Journal}
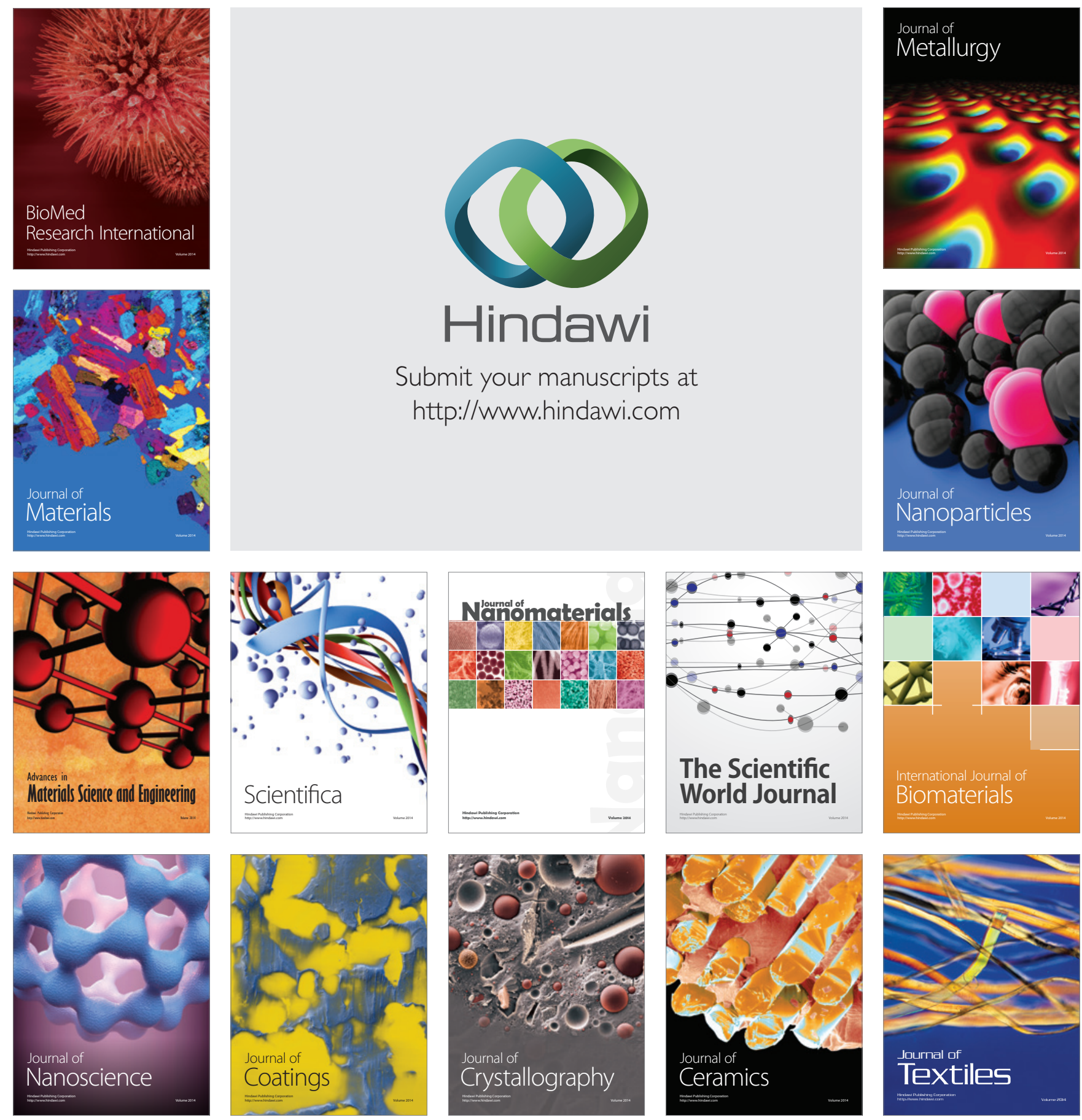